\title{
Caracterização Térmica e Reológica de Borracha de Pneu Desvulcanizada por Microondas
}

\author{
Carlos H. Scuracchio, Daniel A. Waki \\ Departamento de Engenharia de Materiais, FAENQUIL, SP \\ Rosario E. S. Bretas \\ Departamento de Engenharia de Materiais, UFSCar
}

\begin{abstract}
Resumo: Borracha de pneu pós-consumo previamente particulada foi submetida a um tratamento em microondas, de forma a promover a quebra de suas ligações cruzadas, ou seja, sua desvulcanização. A influência das variáveis do processo, tais como tempo de exposição às microondas e número de etapas de tratamento, foi investigada. Após o tratamento, estes materiais foram caracterizados em termos de suas propriedades térmicas por calorimetria exploratória diferencial e reológicas por reometria capilar. Também foi feita extração de solúveis em soxhlet, para avaliar a quantidade de borracha que foi efetivamente desvulcanizada durante o tratamento por microondas. O processo de desvulcanização mostrou-se um método viável para produzir um material passível de ser moldado, de forma semelhante a uma borracha virgem. No entanto, em nenhuma condição de desvulcanização utilizada foi possível obter um material $100 \%$ solúvel, ou seja, totalmente desvulcanizado. A análise térmica sugere que ocorre um processo semelhante ao envelhecimento do material durante o tratamento com as microondas.
\end{abstract}

Palavras-chave: Reciclagem, borracha, desvulcanização, microondas, borracha de pneu moída.

\section{Thermal and Rheological Characterization of Ground Tire Rubber Devulcanized by Microwaves}

Abstract: Ground tire rubber was submitted to a microwaves treatment in order to promote its devulcanization, that is, the breaking of the crosslinks between the chains. The influence of the processing parameters, such as time of exposure to the microwaves and number of treatment steps, was investigated. After the treatment, the material was characterized in terms of its thermal, by DSC, dynamic-mechanical, by DMA and rheological, by capillary rheometry, properties. In addition, the extraction of soluble fractions was made in soxhlet, to evaluate the amount of rubber that effectively was devulcanized during the microwaves treatment. The devulcanization treatment showed to be a viable method to produce a material which can be molded, in a similar way to virgin rubber. However, the complete devulcanization of the material was not possible in any devulcanization condition used. The thermal analysis showed that significant changes in the chemical structure were induced when the material was exposed to the microwaves for long times.

Keywords: Rubber recycling, devulcanization, microwaves, ground tire rubber, GTR.

\section{Introdução}

A reutilização de borrachas vulcanizadas é, atualmente, um dos principais problemas na área de reciclagem ${ }^{[1]}$, por serem termorrígidas, como conseqüência de ligações químicas cruzadas entre as cadeias do polímero, que impedem a fusão e o re-processamento. Dentre as alternativas para o reaproveitamento destes materiais estão a queima e utilização como fonte de energia e combustíveis ${ }^{[2,3]}$, o uso como cargas em materiais para construção civil ${ }^{[4]}$, em polímeros termoplásticos ou termorrígidos ${ }^{[5-8]} \mathrm{e}$ na produção de novos materiais ${ }^{[1]}$. Neste último caso há necessidade de receber algum tipo de tratamento específico que permita a sua re-moldagem. Dentre estes tratamentos encontra-se, por exemplo, a quebra destas ligações cruzadas por processos químico e/ou físico. Esta técnica é chamada de desvulcanização.

Ao contrário do que o nome pode sugerir, a desvulcanização de uma borracha não é simplesmente a reversão do processo de vulcanização, responsável pela formação destas ligações cruzadas. Durante a desvulcanização, uma série de modificações é imposta ao material, o que lhe confere propriedades únicas, diferentes da composição de borracha antes da vulcanização ${ }^{[9,10]}$. Dentre estas modificações pode-se citar: a quebra das ligações cruzadas, normalmente formadas por átomos de enxofre; a quebra de ligações $\mathrm{C}-\mathrm{C}$ e $\mathrm{C}=\mathrm{C}$ da cadeia principal do polímero; a formação de estruturas secundárias na cadeia do polímero, devido à quebra das ligações químicas e o conseqüente aparecimento de radicais livres; a formação de ramificações; e a

Autor para correspondência: Carlos H. Scuracchio, Departamento de Engenharia de Materiais, Faenquil, Rodovia Itajubá-Lorena, km 74,5, CEP: 12600-000, Lorena, SP. E-mail: scuracchio@ppgem.faenquil.br 
diminuição da massa molar do polímero. As modificações na estrutura polimérica dos elastômeros têm um reflexo direto nas propriedades mecânicas e reológicas do material reciclado e ocorrem em maior ou menor grau dependendo do processo de desvulcanização utilizado ${ }^{[11-13]}$.

Devido ao surgimento de novas propriedades no material desvulcanizado torna-se necessário realizar uma caracterização completa destes materiais. Esta caracterização tem dois objetivos principais: i) permitir um conhecimento teórico dos processos que ocorrem durante a desvulcanização, possibilitando, assim, melhorias no processo; ii) ampliar a faixa de possíveis aplicações deste material, assim como ocorre com qualquer material novo.

Dentre as técnicas de desvulcanização descritas na literatura, uma das mais promissoras é a desvulcanização através de microondas ${ }^{[1]}$. Este tipo de processo de desvulcanização tem natureza física, ou seja, não envolve reagentes químicos durante o processo, permitindo a aplicação de alta quantidade de energia ao material em um tempo curto resultando em alta produtividade. Apesar do processo de desvulcanização por microondas ser conhecido desde o final da década de $1970^{[11]}$, a literatura que descreve as propriedades do material desvulcanizado é bastante limitada. Isto tem reduzido tanto o campo de aplicação destes materiais quanto o entendimento do processo de desvulcanização em si.

De forma geral, a desvulcanização através de microondas se dá devido ao aquecimento da amostra, que provoca a quebra das ligações cruzadas em um processo ativado termicamente ${ }^{[12]}$. A situação ideal é que a quantidade de energia absorvida das microondas pela amostra seja elevada o suficiente para quebrar as ligações S-S e C-S das ligações cruzadas, porém não o suficiente para quebrar as ligações C-C e $\mathrm{C}=\mathrm{C}$ da cadeia principal do polímero. Segundo Novotny ${ }^{[11]}$, a energia necessária para a desvulcanização da borracha é de aproximadamente 180 Watt.hora por quilo de borracha. No entanto, para que o material tenha uma boa interação com as microondas e, portanto, um aquecimento eficiente, é necessário que o mesmo apresente uma certa polaridade. Os elastômeros utilizados em pneus são predominantemente misturas físicas de borracha natural (NR) e do copolímero de butadieno-estireno (SBR) ou de NR e polibutadieno (BR). Tanto NR quanto SBR ou BR são polímeros apolares, apresentando quase nenhuma interação com as microondas. Esta dificuldade é contornada pela utilização de cargas condutoras de eletricidade, como o negro de fumo. A presença destas cargas promove um fenômeno chamado de polarização de Maxwell-Wagner ${ }^{[14]}$, o qual permite o aquecimento da borracha de pneu, que tem uma quantidade relativamente alta deste tipo de carga ${ }^{[15]}$.

O objetivo deste artigo é o de descrever as propriedades térmicas e reológicas de borracha de pneu desvulcanizada por microondas sob condições específicas de processo, objetivando tanto entender o processo de desvulcanização em si como as modificações que podem ocorrer com o material durante o tratamento com as microondas e ainda auxiliar na escolha dos melhores parâmetros de processo para uma even-
Tabela 1. Análise granulométrica do pó de borracha Artgoma AG 40

\begin{tabular}{cc}
\hline Malha & $\begin{array}{c}\text { Porcentagem em massa retida } \\
(\boldsymbol{\%})\end{array}$ \\
\hline 20 mesh & 0,67 \\
80 mesh & 80,76 \\
Fundo & 18,57 \\
\hline
\end{tabular}

tual utilização deste material.

\section{Materiais e Métodos}

\section{Material}

Foi utilizada borracha de pneu pós-consumo em pó, gentilmente doada pela Artgoma do Brasil, sob o código AG 40. A análise granulométrica do pó, feita por peneiramento, foi fornecida pela Artgoma e é mostrada na Tabela 1.

A composição exata da borracha, por se tratar de uma mistura de pneus de várias marcas e procedências, é desconhecida; porém, segundo a literatura especializada, a fração de negro de fumo em pneus comerciais é de aproximadamente $31 \%$ em massa ${ }^{[15]}$ e os principais elastômeros são NR, SBR e BR.

\section{Desvulcanização da borracha}

A borracha foi desvulcanizada em um forno de microondas Electrolux modelo 27E, com um magnetron de potência nominal de 700 Watts. De forma a tornar o material tratado mais homogêneo e minimizar o efeito de regiões com alta concentração de onda ("hot spots"), este forno foi adaptado com um sistema de agitação, constituído de uma haste principal com $7 \mathrm{~mm}$ de diâmetro e $100 \mathrm{~mm}$ de comprimento e 8 hastes secundárias com comprimento de $40 \mathrm{~mm}$ e diâmetro de $3 \mathrm{~mm}$, perpendiculares à haste principal. As hastes de agitação foram feitas de aço inoxidável. Este sistema de agitação foi acoplado a um motor com controlador de velocidade. A Figura 1 mostra esquematicamente o sistema utilizado para a desvulcanização das amostras.

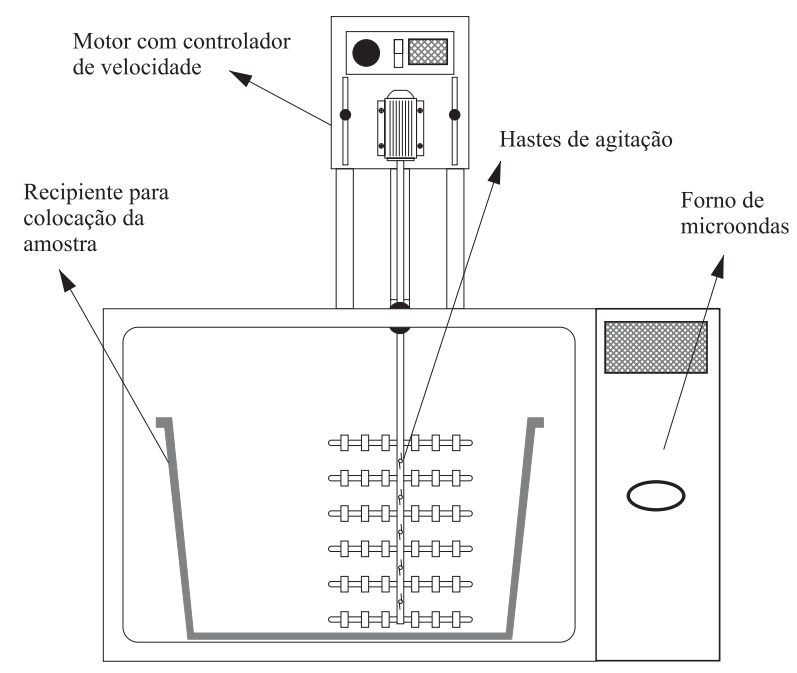

Figura 1. Desenho esquemático do sistema para desvulcanização utilizado. 
Tabela 2. Condições utilizadas no tratamento das amostras de borracha de pneu

\begin{tabular}{lccc}
\hline $\begin{array}{c}\text { Código de } \\
\text { identificação }\end{array}$ & $\begin{array}{c}\text { Tempo de } \\
\text { tratamento para } \\
\text { cada etapa } \\
\text { (min) }\end{array}$ & $\begin{array}{c}\text { Número de } \\
\text { etapas de } \\
\text { tratamento }\end{array}$ & $\begin{array}{c}\text { Tempo de } \\
\text { intervalo entre } \\
\text { etapas de } \\
\text { tratamento } \\
\text { (min) }\end{array}$ \\
\hline GTR 2 & 2 & 1 & - \\
GTR 3 & 3 & 1 & - \\
GTR 4 & 4 & 1 & - \\
GTR 5 & 5 & 1 & 10 \\
GTR 2-2 & 2 & 2 & 10 \\
GTR 2-2-2 & 2 & 3 & 10 \\
GTR 3-3 & 3 & 2 & - \\
\hline
\end{tabular}

O material em pó foi submetido ao tratamento pelas microondas em porções de $60 \mathrm{~g}$, colocadas dentro de um Becker de $120 \mathrm{~mL}$. O sistema de agitação foi ajustado para $40 \mathrm{rpm}$. Foram estudados dois parâmetros de processo: a) tempo de exposições às microondas; b) número de exposições. O tempo de exposição contínuo variou entre 1 e 5 minutos, com intervalos de 1 em 1 minuto. O número de etapas de ataque variou entre 1 e 3 etapas. No caso de mais de uma etapa de tratamento, o material foi deixado resfriando ao ar livre durante 10 minutos.

A Tabela 2 mostra os tipos de tratamentos efetuados e o código de identificação das amostras.

Foram feitas medidas da temperatura de cada material após cada etapa de tratamento através de um termopar do tipo J.

\section{Caracterização do material}

\section{Determinação da quantidade de gel}

A quantidade de gel das borrachas foi determinada em extrator Soxhlet, com tolueno como solvente. Amostras de aproximadamente 5 gramas foram deixadas no extrator em refluxo por 24 horas. Este tempo mostrou-se necessário e suficiente para a extração de todo o material solúvel para as diferentes amostras, já que sua massa não variou depois deste tempo dentro do extrator. Depois da extração completa da parte solúvel, o material juntamente com o filtro foi colocado em uma estufa a $70{ }^{\circ} \mathrm{C}$ por 24 horas para completa evaporação do solvente. Após este período, o conjunto (filtro + amostra) foi pesado. A porcentagem de gel foi, então, calculada pela equação (1):

$$
\% G=\frac{m_{t}-m_{f}}{m_{i}} \times 100
$$

onde:

$\mathrm{m}_{\mathrm{t}}=$ massa do conjunto amostra + papel de filtro após a extração

$\mathrm{m}_{\mathrm{f}}=$ massa do papel de filtro

$\mathrm{m}_{\mathrm{i}}=$ massa inicial da amostra

\section{Características de re-vulcanização do material}

A capacidade do material re-vulcanizar foi testada através da mistura da borracha desvulcanizada com sistema simples de vulcanização, composto de 1,3 phr do acelerador de vulcanização N-ciclohexil-2-benzotiazola sulfenamida e $2 \mathrm{phr}$ de enxofre. A mistura foi feita em um moinho de rolos para laboratório Cope. O moinho é constituído de dois cilindros de diâmetro $20 \mathrm{~cm}$ e $40 \mathrm{~cm}$ de comprimento e com razão de velocidade de rotação entre os cilindros de 18/22. A mistura foi feita por aproximadamente 5 minutos. Para acompanhar a vulcanização, foi utilizado um reômetro de disco oscilatório Monsanto, de acordo com a norma ASTM D2084. O resultado foi apresentado na forma de torque versus tempo em temperatura de $180{ }^{\circ} \mathrm{C}$. A duração total do ensaio foi de 12 minutos.

\section{Calorimetria Exploratória Diferencial}

A caracterização térmica por calorimetria exploratória diferencial foi realizada principalmente para detecção da temperatura de transição vítrea $\left(\mathrm{T}_{\mathrm{g}}\right)$ e do comportamento de degradação, e foi feita em um DSC QA 100 da TA Instruments. A taxa de aquecimento escolhida foi de $10{ }^{\circ} \mathrm{C} / \mathrm{min}$, a massa de amostra foi de aproximadamente $8 \mathrm{mg}$ e as análises foram executadas em atmosfera inerte de nitrogênio. Os resultados foram apresentados na forma de fluxo de calor versus temperatura.

\section{Reometria Capilar}

As medidas de viscosidade em função da taxa de cisalhamento dos materiais desvulcanizados foram feitas em um reômetro capilar Instron 4467, com capilar de diâmetro $1,27 \mathrm{~mm}$ e $\mathrm{L} / \mathrm{D}=40$. A velocidade do pistão foi ajustada de forma a gerar taxas de cisalhamento no capilar entre $40 \mathrm{e}$ $3000 \mathrm{~s}^{-1}$. A temperatura de ensaio foi de $180^{\circ} \mathrm{C}$.

\section{Resultados e Discussão}

\section{Medidas de temperatura do material após a desvulcanização}

A Tabela 3 mostra os resultados de medidas de temperatura após cada etapa de tratamento das borrachas.

Tabela 3. Temperatura após cada etapa de tratamento da borracha de pneu

\begin{tabular}{lccc}
\hline Amostra & $\begin{array}{c}\text { T no 1 } \\
\text { intervalo } \\
\left({ }^{\circ} \mathbf{C}\right)\end{array}$ & $\begin{array}{c}\text { T no 2 } \\
\text { intervalo } \\
\left({ }^{\circ} \mathbf{C}\right)\end{array}$ & $\begin{array}{c}\text { T no 3 } \\
\text { intervalo } \\
\left({ }^{\circ} \mathbf{C}\right)\end{array}$ \\
\hline GTR 1 & 95 & - & - \\
GTR 2 & 141 & - & - \\
GTR 3 & 236 & - & - \\
GTR 4 & 282 & - & - \\
GTR 5 & 312 & - & - \\
GTR 2-2 & 145 & 202 & - \\
GTR 2-2-2 & 130 & 182 & 204 \\
GTR 3-3 & 193 & 302 & - \\
\hline
\end{tabular}


Tabela 4. Porcentagem em gel das amostras de GTR

\begin{tabular}{lc}
\hline \multicolumn{1}{c}{ Amostra } & $\begin{array}{c}\text { Quantidade de gel } \\
(\%)\end{array}$ \\
\hline GTR conforme recebido & 85 \\
GTR 2 & 84 \\
GTR 3 & 64 \\
GTR 4 & 54 \\
GTR 5 & $*$ \\
GTR 2-2 & 74 \\
GTR 2-2-2 & 72 \\
GTR 3-3 & 56 \\
\hline
\end{tabular}

*) Amostra totalmente degradada

Pode-se observar que, conforme esperado, quanto maior o tempo de tratamento maior a temperatura final do material. O maior tempo de tratamento utilizado foi de 5 minutos. A exposição da borracha às microondas por tempos iguais ou maiores que 5 minutos produziu material completamente degradado, na forma de um pó muito semelhante a negro de fumo ou carvão.

\section{Medidas de quantidade de gel}

A Tabela 4 mostra a quantidade de gel medido por extração em Soxhlet para as amostras vulcanizadas e desvulcanizadas.

A quantidade relativa de gel (material insolúvel) e de sol (material solúvel) é uma medida direta do tratamento causando envelhecimento e/ou de desvulcanização. Uma maior quantidade de sol e menor quantidade de gel significa que houve modificação das cadeias poliméricas podendo ser relacionada também com rompimento da rede tridimensional formada pela reticulação ${ }^{[16]}$. Da Tabela 4 pode-se observar que a quantidade de gel diminuiu com o aumento do tempo de tratamento em microondas. Também há uma diminuição da quantidade de gel com o aumento da quantidade de etapas de tratamento. Este comportamento era de se esperar, já que quanto maior o tempo de tratamento, maior a temperatura alcançada pelo material e maior a extensão da desvulcanização. O fato de haver uma parte solúvel em tolueno mesmo para a amostra antes do tratamento pelas microondas provavelmente é devido à presença de óleos auxiliares de processo na borracha. Pode-se observar, também, que o tempo de tratamento tem uma maior influência na quantidade de gel que a quantidade de ciclos de tratamento.

\section{Características de re-vulcanização}

A Figura 2 mostra as curvas de re-vulcanização das borrachas.

As amostras GTR 4 e GTR 5 não puderam ser compostas no moinho de rolos; no caso do GTR 4 por este ser muito pegajoso, e no caso do GTR 5, porque a borracha foi totalmente degradada, tornando-se um pó. Quanto à amostra GTR 2, também não foi possível fazer a mistura porque não houve desvulcanização aparente, e a amostra não apresentou

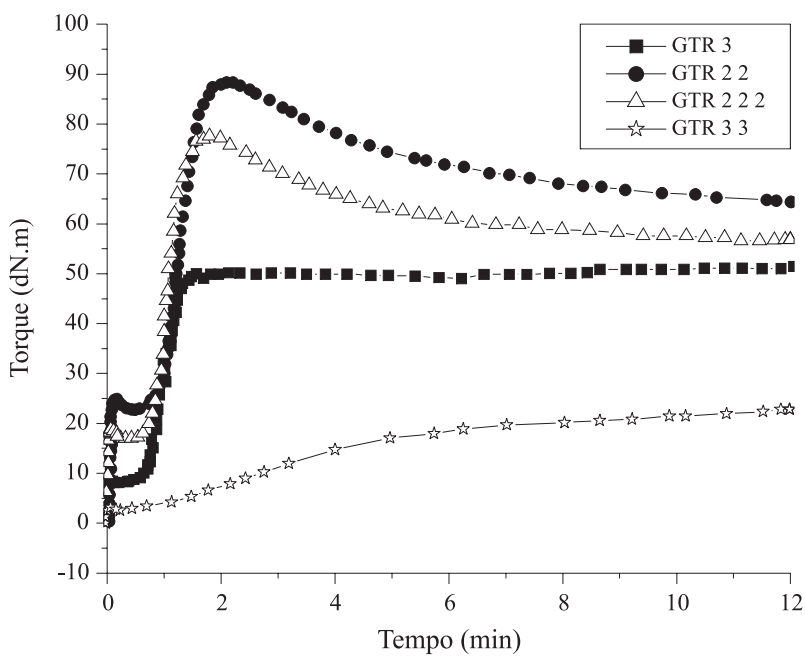

Figura 2. Curvas de re-vulcanização das borrachas desvulcanizadas

homogeneidade na mistura com os agentes de vulcanização.

Pode-se observar, das curvas da Figura 2, que as amostras GTR 3, GTR 2-2 e GTR 2-2-2 apresentaram tempos de prévulcanização ("scorch-time") praticamente iguais de aproximadamente 1 minuto. Estas amostras também mostraram uma capacidade de re-vulcanização bastante acentuada, porém houve uma reversão na cura ou degradação da cadeia polimérica a partir de 2 minutos a $180^{\circ} \mathrm{C}$. Este comportamento de reversão é observado normalmente em borracha natural é a borracha em maior proporção na mistura e tem natureza química facilmente envelhecida pelo calor. Pode-se observar também que quanto menor a quantidade de gel, menor o torque final, ou seja, menor a rigidez da borracha. Este fato está ligado, provavelmente, a um dos mecanismos de degradação da cadeia do polímero, comprovados neste trabalho nos experimentos com tempos de exposição às microondas superiores a 5 minutos. Um caso crítico foi da amostra GTR 3-3 que apresentou um torque muito abaixo das outras amostras, não chegando, nem mesmo, a apresentar uma vulcanização completa.

A diminuição da rigidez da borracha pode ser um problema, já que a maioria das aplicações da borracha exige um módulo de rigidez alto. Desta forma, um maior ataque das microondas ao material pode ser apropriado para melhorar sua processabilidade, porém é necessário ter em mente que isso pode limitar sua utilização final, devido à perda em suas propriedades. A Tabela 5 apresenta os valores de tempo de pré-cura $\left(\mathrm{t}_{\mathrm{S} 2}\right)$, tempo ótimo de vulcanização $\left(\mathrm{t}_{\mathrm{C} 90}\right)$ e torque máximo apresentado por cada amostra.

Tabela 5. Valores de $\mathrm{t}_{\mathrm{S} 2}, \mathrm{t}_{\mathrm{C} 90}$ e torque máximo.

\begin{tabular}{lccc}
\hline Amostra & $\begin{array}{c}\mathbf{t}_{\mathbf{c 9 0}} \\
(\mathbf{m i n})\end{array}$ & $\begin{array}{c}\mathbf{t}_{\mathbf{s 2}} \\
(\mathbf{m i n})\end{array}$ & $\begin{array}{c}\text { Torque máximo } \\
(\mathbf{d N . m})\end{array}$ \\
\hline GTR 3 & 1,25 & 0,55 & 50 \\
GTR 2-2 & 1,50 & 0,60 & 80 \\
GTR 2-2-2 & 1,30 & 0,65 & 70,0 \\
GTR 3-3 & 8,30 & 1,30 & 23 \\
\hline
\end{tabular}


Pode-se observar que, de forma geral, o aumento no tempo de tratamento teve pouca influência nos valores de tempo de pré cura $t_{\mathrm{C} 90}$ e $t_{\mathrm{S} 2}$, indicando não haver uma influência significativa do tempo de tratamento na cinética de re-vulcanização do material. Este dado é de extrema importância no processamento do material, já que a vulcanização é a etapa que mais consome tempo na fabricação de artigos de borracha. Uma exceção é o GTR 3 3, que teve os valores de $\mathrm{t}_{\mathrm{C} 90}$ e $\mathrm{t}_{\mathrm{S} 2}$ bem acima das outras borrachas. Dentre as possíveis explicações para este fato está a degradação muito intensa das cadeias do polímero, que, conforme já discutido anteriormente, gerou como resultado um torque muito baixo.

\section{Calorimetria Exploratória Diferencial, DSC}

A Figura 3 mostra as curvas de calorimetria exploratória diferencial para as amostras que foram tratadas em uma única etapa. As amostras tratadas em mais de uma etapa apresentaram formatos da curva de calorimetria exploratória diferencial semelhantes.

As curvas da Figura 3 apresentam duas transições térmicas principais: a primeira, onde ocorre uma mudança na declividade da curva e conseqüentemente no calor específico da amostra é associada à $\mathrm{T}_{\mathrm{g}}$ do material; a segunda, onde ocorre um pico exotérmico, corresponde à degradação do material. Estas temperaturas são mostradas na Tabela 6.

A amostra GTR 5 não mostrou nem a presença de picos de degradação, nem a inflexão da curva típica de $\mathrm{T}_{\mathrm{g}}$, devido à amostra ter sido seriamente degradada durante o tratamento com microondas, tornando-se um pó semelhante ao negro de fumo. Não houve variação significativa no valor de $T_{\mathrm{g}}$ para as amostras sem tratamento, GTR 2, GTR 3, GTR 2-2 e GTR 2-2-2. No entanto, pode-se verificar um aumento significativo da $\mathrm{T}_{\mathrm{g}}$ das amostras GTR 4 e GTR 3-3. Verificando-se a quantidade de gel e as curvas de torque em reômetro Monsanto, pode-se observar que estas amostras foram as mais severamente atacadas pela ação das microondas, ou seja, são as que sofreram uma maior quantidade de desvulcanização. Isto leva a supor que algum tipo de modificação na estrutura química do material, induzida pelo processo de desvulca-

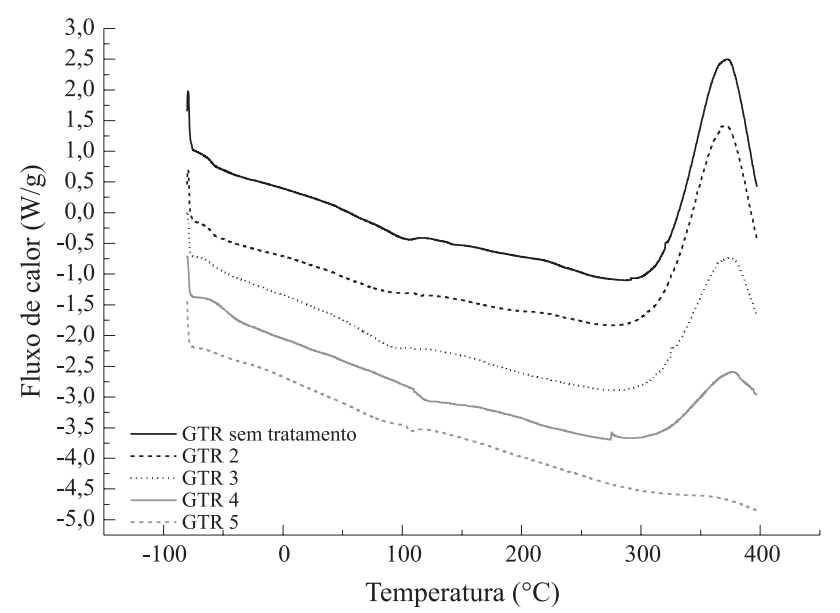

Figura 3. Resultados da análise calorimétrica exploratória diferencial das amostras tratadas em uma única etapa
Tabela 6. Temperaturas de transição vítrea e de degradação das amostras

\begin{tabular}{lcc}
\hline \multicolumn{1}{c}{ Amostra } & $\begin{array}{c}\mathbf{T}_{\mathbf{g}} \\
\left({ }^{\circ} \mathbf{C}\right)\end{array}$ & $\begin{array}{c}\text { Temperatura de degradação } \\
\left({ }^{\circ} \mathbf{C}\right)\end{array}$ \\
\hline GTR sem tratamento & $-59,7$ & 372 \\
GTR 2 & $-59,6$ & 371 \\
GTR 3 & $-60,1$ & 375 \\
GTR 4 & $-46,8$ & 377 \\
GTR 5 & nd & nd \\
GTR 2-2 & $-61,4$ & 375 \\
GTR 2-2-2 & $-62,1$ & 374 \\
GTR 3-3 & $-56,7$ & 377 \\
\hline
\end{tabular}

nd: não detectado

nização, ocorreu. $\mathrm{O}$ aumento da $\mathrm{T}_{\mathrm{g}}$ em amostras desvulcanizadas por ultra-som ${ }^{[9]}$ é atribuído à formação de estruturas cíclicas de enxofre na cadeia principal do polímero. Estas estruturas cíclicas tendem a diminuir a mobilidade segmental da cadeia do polímero e aumentar o valor de $\mathrm{T}_{\mathrm{g}}$, e são provenientes da quebra de ligações cruzadas polissulfídicas, que são as mais sensíveis à influência de calor.

A temperatura de degradação, conforme detectada pelo DSC, não foi afetada significativamente pelo tratamento em microondas. Para todas as amostras, o pico máximo de degradação ocorreu entre $370^{\circ} \mathrm{C}$ e $375^{\circ} \mathrm{C}$, porém o início das reações de degradação, nas condições utilizadas na análise de DSC, ficou em aproximadamente $300{ }^{\circ} \mathrm{C}$. Houve uma diferença na altura dos picos de degradação da borracha com o tempo de exposição às microondas. Este fato era de se esperar, já que quanto mais agressivo o ataque, maior a degradação da borracha durante o ataque com as microondas e menor quantidade de polímero para ser degradado durante o ensaio de DSC.

\section{Reometria Capilar}

A Figura 4 mostra os resultados de viscosidade em função da taxa de cisalhamento dos materiais.

Não foi possível realizar os ensaios para as amostras de GTR conforme recebida e com 2 minutos de tratamento em microondas, devido ao travamento das amostras dentro do equipamento (amostras sólidas).

A Figura 4 mostra que as curvas de viscosidade das borrachas podem ser representadas pela Lei das Potências, conforme a seguinte equação :

$$
\tau=m \dot{\gamma}^{n}
$$

onde: $\mathrm{m}=$ consistência

$\mathrm{n}=$ índice de potências

$\tau=$ tensão de cisalhamento

$\dot{\gamma}=$ taxa de cisalhamento

Os valores de $\mathrm{m}$ e $\mathrm{n}$ calculados são mostrados na Tabela 7.

Pode-se observar que o valor de índice de potências não variou muito com o tipo de tratamento sofrido pelas amostras, com exceção do GTR 4, que apresentou um comportamento mais pseudoplástico. Já o valor de consistência teve 


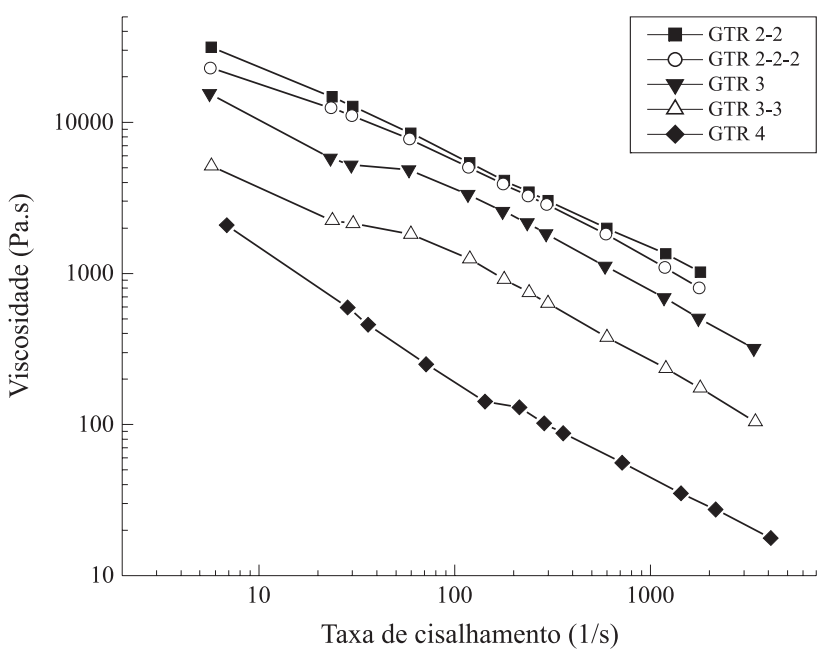

Figura 4. Curvas de viscosidade versus taxa de cisalhamento a $180{ }^{\circ} \mathrm{C}$ para as amostras de GTR desvulcanizadas

Tabela 7. Valores de m e $\mathrm{n}$ para as amostras de GTR desvulcanizadas.

\begin{tabular}{ccc}
\hline Amostra & m $\left(\mathbf{P a . S}^{\mathbf{n}}\right)$ & $\mathbf{n}$ \\
\hline GTR 2-2 & 107150 & 0,40 \\
GTR 2-2-2 & 89125 & 0,41 \\
GTR 3 & 51286 & 0,41 \\
GTR 3-3 & 20417 & 0,39 \\
GTR 4 & 7413 & 0,28 \\
\hline
\end{tabular}

uma queda significativa com o tempo de tratamento para todas as amostras de GTR. Isto significa que quanto maior o tempo de tratamento das amostras, menor sua viscosidade em todas as taxas de cisalhamento analisadas indicando uma menor massa molar média corroborando os resultados anteriores sobre o envelhecimento e degradação da cadeia pelas microondas. Estes resultados estão de acordo com os resultados de quantidade de gel e de reometria de torque em reômetro Monsanto, ou seja, quanto maior a quantidade de desvulcanização sofrida pela amostra, menor a viscosidade da mesma e provavelmente menor a massa molar do polímero. Em termos de processamento, a diminuição da viscosidade representa uma menor quantidade de energia necessária para o fluxo do material e maior facilidade de processamento. $\mathrm{O}$ controle da viscosidade deve ser feito uma vez que está diretamente relacionado ao desempenho mecânico do material.

O menor valor do índice de potências da amostra GTR 4 pode ser explicado pela distribuição de massa molar do polímero após o tratamento. Esta foi a amostra que sofreu o ataque mais agressivo, portanto é de se esperar que a mesma tenha sofrido uma maior degradação das cadeias poliméricas. Esta maior degradação acaba por se manifestar numa distribuição de massa molar mais larga, com a presença de cadeias moleculares curtas. Estas cadeias curtas podem ser responsáveis pela maior capacidade de desemaranhamento das moléculas durante o fluxo, funcionando como um plastificante e diminuindo o valor de $\mathrm{n}^{[17]}$.

\section{Conclusões}

A técnica de desvulcanização por microondas foi capaz de gerar um material com propriedades bastante diferentes da borracha vulcanizada original. Dentre as propriedades, a que de maior destaque, no que se refere à possibilidade do material ser reciclado, é a capacidade de fluir e ser moldado. Esta característica, juntamente com a capacidade que o material tem de ser re-vulcanizado, aponta para a grande aplicabilidade da desvulcanização do material por microondas.

A desvulcanização pode ser considerada como inversamente proporcional à quantidade de gel do material resultante. Uma maior eficiência do processo de desvulcanização provoca uma maior moldabilidade do material, melhorando suas propriedades de moldagem. No entanto, quanto maior a desvulcanização, maior a quantidade de ligações da cadeia principal do polímero que são quebradas. O efeito desta degradação do polímero é uma redução significativa da rigidez do material quando re-vulcanizado. Dependendo do tipo de utilização do material recuperado, isto pode dificultar o seu uso. Portanto, a escolha dos parâmetros do processo de desvulcanização deve levar em conta o balanço entre processabilidade e propriedades mecânicas que se deseja do material final.

A análise térmica dos materiais também sugere que ocorre envelhecimento do polímero depois de um certo tempo de tratamento. Estas diferenças estruturais seriam responsáveis pela mudança na temperatura de transição vítrea observada nos materiais que tiveram tempos de exposição às microondas maiores.

\section{Agradecimentos}

Os autores gostariam de agradecer à FAPESP, processo 03/08175-2, pelo apoio financeiro.

\section{Referências Bibliográficas}

1. Adhikari, B. and Maiti, de D. - "Reclamation and recycling of waste rubber"; Progress in Polymer Science, Vol. 25, pags. 909-948, (2000).

2. Sharma, V. K.; Fortuna, F.; Mincarini, M.; Berillo, M. and Cornacchia, G. - "Disposal of waste tyres for energy safe environment"; Applied Energy, Vol. 65. pags. 381394, (2000).

3. Chang, Y., M. - "On pyrolysis of waste tire: degradation rate and product yields"; Resources, Conservation and Recycling, Vol. 17, pags. 125-139, (1996).

4. Siddique, R.; Naik, T. R. - "Properties of concrete containing scrap-tire rubber - an overview"; Waste Management Vol. 24, pags. 563-569, (2004).

5. Pittolo, M.; Burford, R. P. - "Recycled Rubber Crumb as a Toughener or Polystyrene"; Rubber Chemistry and Technology, Vol. 58, pags. 97-107, (1985). 
6. Araújo, E. M.; Carvalho, A. H.; Fook, M. V.L. and D'almeida, J. R. M. - "Propriedades Mecânicas de blendas de PS/ resíduo de borracha - Influência da concentração, granulometria e método de moldagem"; Polímeros: Ciência e Tecnologia, Jul/Set 1997, pags. 45-52, (1997).

7. Oliphant, K. and Baker, W. E. - "The Use of Cryogenically Ground Rubber Tires as a Filler in Polyolefin Blends"; Polymer Engineering and Science, Vol. 33, No. 3, (1993).

8. Scuracchio, C. H.; Bretas, R. E. S. and Isayev, A. I. - "Blends of PS with SBR Devulcanized By Ultrasound: Rheology and Morphology"; Journal of Elastomers and Plastics, Vol. 36, pags. 45-75, (2004).

9. Levin, V. Y.; Kim, S. H.; Isayev, A.i.; Von Meerwall, E. and Massey, J. - "Ultrasound Devulcanization of Sulphur Vulcanized SBR: Crosslink Density and Molecular Mobility"; Rubber Chemistry and Technology, Vol. 69, pags. 104-103, (1996).

10. Levin, V. Y.; Isayev, A.i. and Kim, S. H. - "Superior mechanical properties of reclaimed SBR with bimodal network"; Rubber Chemistry and Technology, Vol. 70, pags. 194-201, (1997).

11. Novotny, D. S.; Marsh, R. I.; Masters, F. C. and Tally, D. N. - "Microwave devulcanization of rubber"; Patente No. US 4,104,205, (1978).

12. Wicks, G. G.; Schulz, R. I.; Clark, D. E. and Folzd. C. "Microwave treatment of vulcanized rubber"; Patente No. 6,420,457, (2002).
13. Masaki, K.; Ohkawara, S. I.; Hirano, T.; Seno, M. and Sato, T. - "Devulcanization of nitrile butadiene rubber in nitrobenzene"; Journal of Applied Polymer Science, Vol. 91, pags. 3342-3353, (2004).

14. Tostenson, E. T. and Chou, T. W. - "Microwave processing, fundamentals and applications"; Composites: Part A: Applied Science and Manufacturing", Vol. 30, pags. 1055-1071, (1999).

15. Amari T.; Themelis, N. J. and Wernick, K. W. - "Resource recovery from used rubber tires" Resources Policy, Vol. 25, pags. 179-188, (1999).

16. Yashin, V. V. and Isayev, A. I. - "A Model for Rubber Degradation Under Ultrasonic Treatment: PartII. Rupture of Rubber Network and Comparison with Experiments"; Rubber Chemistry and Technology, Vol.73, pags. 325-339, (2000).

17. Bretas, R. E. S. and Ávila, M. A. - "Reologia de Polímeros Fundidos".

18. Turi, E. A. - "Thermal characterization of polymeric materials"; 2ed. San Diego, Academic Press, pág. 264, 1997.

19. Canevarolo Jr., S. V. - "Análise térmica dinâmicomecânica", em Técnicas de Caracterização de Polímeros, $1^{\text {a }}$ Edição, Ed. Artliber, São Paulo, (2004).

Enviado: 07/04/05

Reenviado: 13/07/05

Aprovado: 26/08/05 